\title{
Survival of male patients with spinal cord injury after cardiac arrest in Department of Veterans Affairs hospital: Pilot study
}

\author{
Deborah Caruso, MD; ${ }^{1}$ William E. Carter, MD/MPH; ${ }^{1-2 *}$ David X. Cifu, MD; ${ }^{2-3}$ William Carne, PhD $^{\mathbf{4}}$ \\ ${ }^{1}$ Spinal Cord Injury and Disorders Center of Excellence, Hunter Holmes McGuire Department of Veterans Affairs \\ Medical Center (VAMC), Richmond, VA; ${ }^{2}$ Department of Physical Medicine and Rehabilitation, Virginia Common- \\ wealth University, Richmond, VA; ${ }^{3}$ Physical Medicine and Rehabilitation Services, Veterans Health Administration, \\ Washington, DC; ${ }^{4}$ Neurology Service, Hunter Holmes McGuire VAMC, Richmond, VA
}

\begin{abstract}
Survivability characteristics after cardiopulmonary resuscitation in the population with spinal cord injury (SCI) are unclear but may be useful for advanced care planning discussions with patients. Retrospective evaluation from records of all SCI patients over $10 \mathrm{yr}$ at a Department of Veterans Affairs medical center who experienced in-hospital cardiac arrest was performed. Demographic data and other common measurements were recorded. Thirty-six male subjects were identified, and only two patients survived to discharge $(5.5 \%$ survival rate), both of whom were admitted for nonacute issues and were asymptomatic shortly before the cardiac arrest. The mean age at the time of cardiopulmonary arrest was $62.4 \mathrm{yr}$, with a mean time from cardiac arrest to death of $3.02 \mathrm{~d}$. No significant demographic parameters were identified. Overall, SCI likely portends worse outcome for acutely ill patients in the situation of a cardiac arrest. Conclusions are limited by sample size.
\end{abstract}

Key words: advanced care planning, cardiac arrest, cardiopulmonary resuscitation, CPR, inpatient, SCI, spinal cord injury, survival, VA hospitals, Veteran population.

\section{INTRODUCTION}

Cardiopulmonary resuscitation (CPR) was initially developed in the 1960s to treat patients with sudden, unexpected cardiopulmonary failure [1]. Subsequently, CPR usage has spread worldwide and is the standard of care for the acute management of cardiac arrest, including in the hospitalized setting, unless a "do not resuscitate" (DNR) order has been designated. Survival rates after inhospital cardiac arrest range from 0 to 44 percent, with a 15-20 percent mean rate [2]. Of interest, no published studies have examined the survivability after cardiac arrest in patients with spinal cord injury (SCI), a condition with multiple life-threatening comorbidities. For medical professionals working with any high-risk patient population, including patients with SCI, advanced care planning (ACP) involves educating patients on decisions that will need to be made in the event of a severe, life-threatening acute illness and then helping patients communicate their wishes. In many circumstances, however, patients and families misunderstand the role of ACP and avoid appropriate planning, thereby making uninformed decisions during urgent situations, such as cardiopulmonary arrest

\footnotetext{
Abbreviations: $\mathrm{ACP}=$ advanced care planning, $\mathrm{BMI}=$ body mass index, $\mathrm{CPR}=$ cardiopulmonary resuscitation, $\mathrm{DNR}=$ do not resuscitate, $\mathrm{ICU}=$ intensive care unit, $\mathrm{SCI}=$ spinal cord injury, SD = standard deviation.

*Address all correspondence to William E. Carter, MD/ MPH; Virginia Commonwealth University-Physical Medicine and Rehabilitation, PO Box 980661, Richmond, VA 23298; 804-828-0862; fax: 804-828-5074.

Email: willecarter@gmail.com

http://dx.doi.org/10.1682/JRRD.2013.05.0119
} 
[3]. When next of kin is unavailable, physicians are inaccurate $\sim 50$ percent of the time when predicting patient preferences in cardiac arrest situations [4].

Moss reviewed 19 major studies of the nondisabled population and documented a 14 percent average survival rate for in-hospital CPR, with a 5.3-18.5 percent survival rate 6 mo postdischarge [5]. The best survival rates were associated with myocardial infarction-induced arrhythmias (26\%-46\%), ventricular arrhythmia associated with congestive heart failure (19\%-50\%), and drug overdose/ reaction $(22 \%-28 \%)$. The poorest survival rates $(<10 \%)$ were consistently associated with malignancies, neurological disease, renal failure, and sepsis with survival rates. Survival was not associated with sex, age, or where in the hospital the arrest occurred [5].

A number of studies have examined the effect of demographics on survivability after cardiac arrest in the nondisabled population. In a study of 28,389 cardiac arrests, the mean age of arrest was $65.9 \mathrm{yr}$ [6]. African Americans have been shown to have lower hospital discharge rates after CPR [7]. In a review of 21,237 adults, individuals with a body mass index (BMI) $<30$ or $>35$ had lower rates of discharge with shockable rhythms than did those with a BMI between 30 and 34.9 [8]. Additionally, patients with a BMI $<18.5$ were less likely to survive a nonshockable rhythm than were patients with a BMI $\geq 18.5$. Importantly, a 63.4 percent decrease in discharge functional status has been demonstrated in survivors of CPR compared with other non-CPR discharges [9] and one-third of in-hospital CPR survivors wish that CPR had not been performed [7].

Survivability information for the SCI population would be useful for ACP by both patients and clinicians. Individuals with acute and chronic SCI have a number of elevated risks for cardiopulmonary arrest, including deep venous thrombosis, mucous plugging, autonomic dysreflexia [10], and cardiomyopathy; however, no studies have investigated rates of hospital survival after cardiopulmonary resuscitation in this population. The same factors that put many of these individuals at risk for cardiac arrest could also be expected to reduce the success of CPR in this group. The goals of this pilot investigation were to better characterize the prevalence of sudden inhospital death and the survivability after CPR in a large SCI population over a $10 \mathrm{yr}$ period. This information will have utility for both clinicians and patients to better inform ACP and end-of-life decisions.

\section{METHODS}

\section{Subjects}

Records for all consecutive SCI patients admitted to the Hunter Holmes McGuire Department of Veterans Affairs Medical Center from January 1, 2002, through January 1, 2012, who experienced in-hospital cardiac arrest on the SCI unit were retrospectively reviewed. These data were provided by the regional Veterans Integrated Service Network Data Warehouse site manager as screened by documentation of a "code blue" note in the chart. The presence of true cardiac arrest was then verified through chart review, with cardiac arrest defined as a sudden cessation of heart function including pulseless electrical activity, asystole, and ventricular fibrillation. Data elements extracted from the medical record for each patient included demographic data, duration of disease, neurological level defined by American Spinal Injury Association classification, BMI, status of survival, hospital day number, admission diagnosis, and discharge disposition. Several additional variables were obtained to try to characterize and assess illness severity before the cardiac arrest. "Acutely ill at time of cardiac arrest" was defined by acute worsening of laboratory values, vital signs, intensive care unit (ICU) location status, or notation from any provider of concern for decompensation in the $5 \mathrm{~d}$ before the cardiac arrest. Verification of prior ACP was assessed as well.

\section{Statistical Analysis}

Statistical analyses were performed using SPSS 14.0 (IBM Corporation; Armonk, New York) to calculate frequencies, means, ranges, and standard deviations (SDs) as applicable. Pearson product moment correlations were also performed to examine relationships between various variables. The small sample size precluded meaningful testing for between-group mean differences.

\section{RESULTS}

Complete demographic data are displayed in Table 1. Of a total of 5,290 patients admitted to the SCI unit during the $10 \mathrm{yr}$ study period, $36(0.6 \%)$ experienced in-hospital cardiac arrest. All 36 subjects were male, with a mean age of $62.5 \mathrm{yr}$ (range $=40-91$ ) and a mean BMI of 24.1. Regarding race, 13 (36.1\%) of the subjects were African American, 13 (36.1\%) were Caucasian, and 10 (27.8\%) were of unknown/undeclared race. The SCI lesion level 
Table 1.

Demographic variables for 36 male patients with spinal cord injury (SCI) who experienced in-hospital cardiac arrest.

\begin{tabular}{|c|c|c|c|c|}
\hline Variable & $n$ & Mean & $\%$ & SD \\
\hline Age & 36 & 62.5 & - & 10.8 \\
\hline Weight (lb) & 35 & 178 & - & 43.0 \\
\hline BMI & 25 & 24.1 & - & 5.9 \\
\hline \multicolumn{5}{|l|}{ Race } \\
\hline Caucasian & 13 & - & 36 & - \\
\hline African American & 13 & - & 36 & - \\
\hline \multicolumn{5}{|l|}{ AIS } \\
\hline A & 28 & - & 78 & - \\
\hline B & 6 & - & 17 & - \\
\hline $\mathrm{C}$ & 2 & - & 6 & - \\
\hline $\mathrm{D}$ & 0 & - & 0 & - \\
\hline \multicolumn{5}{|l|}{ Chronic Conditions ${ }^{*}$} \\
\hline Cancer & 6 & - & 17 & - \\
\hline End Stage Renal Disease & 8 & - & 22 & - \\
\hline Diabetes & 16 & - & 44 & - \\
\hline Cardiovascular Disease & 16 & - & 44 & - \\
\hline COPD/Tracheostomy & 4 & - & 11 & - \\
\hline Pressure Ulcer & 25 & - & 69 & - \\
\hline
\end{tabular}

was cervical in 22 (61\%) of the subjects and thoracic in 14 (39\%); 34 (94\%) of the subjects had motor complete injuries and 28 (78\%) had motor and sensory complete injuries. Of the 36 patients, 6 had cancer (excluding skin cancers), 8 had kidney disease, 16 were diabetic, 16 had cardiovascular disease, and 4 had pulmonary conditions. Of these five chronic condition classifications, 25 percent of subjects had three or more. Only two subjects had none of these conditions but had complications from surgical procedures.

None of the subjects was admitted for their primary SCI rehabilitation and only five of the subjects were less than $1 \mathrm{yr}$ from their initial injury (Table 2). Twelve (33.3\%) subjects had decubitus ulcers as a major medical reason for admission; four (11.1\%) subjects were convenience admissions for routine laboratory work or testing because of distance of home from hospital; and the remainder were admitted for various other medical care issues, such as elective cystoscopy, failure to thrive, fecal impaction, deep vein thrombosis, end stage renal disease, and thoracic osteomyelitis. Length of hospitalization from day of admission until cardiac arrest ranged from a few days to nearly 19 mo (mean $=91.8, \mathrm{SD}=109.7$ ).

Two-thirds of the patients met criteria for being acutely ill at the time of the cardiac arrest (defined as fever, abnormal vitals, abnormal laboratory values, ICU or medicine service transfer, or noted to be unstable or other concerns reflected in the chart by hospital staff), and nearly 20 percent were already in the ICU before cardiac arrest. Intubation occurred during every cardiac arrest. Of the 36 patients, 28 (78\%) had chart documentation indicating that advanced care directives had been explained by staff. Eighty-nine percent declined consent to a DNR order, while four consented to a DNR. Interestingly, three of the patients who initially consented later rescinded consent.

Of the 36 patients, only 2 survived and were later discharged to their previous living environment (survival rate of 5.5\%). For the remainder, the mean time from resuscitation to death was $3.02 \mathrm{~d}$. All patients were transferred to 
JRRD, Volume 51, Number 7, 2014

Table 2.

Clinical variables for 36 male patients with spinal cord injury (SCI) who experienced in-hospital cardiac arrest.

\begin{tabular}{lcccc}
\hline \multicolumn{1}{c}{ Variable } & n & Mean & \% & SD \\
\hline Mortality (d) & 34 & 3 & 94.4 & 7 \\
Mortality Timing & & & & - \\
Day of Cardiac Arrest & 23 & - & 64.0 & - \\
Before Discharge & 11 & - & 36.0 & - \\
SCI-Injured Years Before Cardiac Arrest & & 26 & 14.0 & - \\
$<1$ & 5 & - & 11.0 & - \\
$1-2$ & 4 & - & 75.0 & - \\
$>2$ & 27 & & & - \\
Reason for Admission & & - & 33.0 & - \\
Nonacute & 3 & - & 58.0 & - \\
Decubitus Ulcer-Related & 12 & 91.8 & & - \\
Other Acute Reason & 21 & - & 66.7 & - \\
Hospital Day No. & 36 & - & 19.4 & - \\
Acutely Ill at Time of Cardiac Arrest & 34 & - & 19.4 & \\
ICU at Time of Cardiac Arrest & 7 & &
\end{tabular}

the ICU after cardiac arrest. All deaths occurred in the ICU. There were no significant Pearson correlation coefficients between time from injury to cardiac arrest and either weight, BMI, or age. Of the survivors, one had cardiac arrest secondary to opiate overdose. The other's arrest occurred for unclear reason, but he was admitted for an annual SCI evaluation, was asymptomatic before the arrest, and had a history of mucous plugging. One survivor was alive at the time of the study's completion, but the other died $1 \mathrm{yr}$ after cardiac arrest from malignancy. For those who survived the first day but died in-hospital (36\% of subjects), the average survival time was $11 \mathrm{~d}$. None regained enough stability to be transferred from the ICU. Statistical analysis did not yield differences in demographics between those surviving the initial cardiac arrest and nonsurvivors.

\section{DISCUSSION}

This pilot study was initiated to investigate survival rates from CPR in a Veteran SCI population over a $10 \mathrm{yr}$ period. As expected, we found a poor survival rate of 5.5 percent, which was about one-third the reported rate for general populations. This study represents the first published investigation of the rates and survivability of in-hospital sudden death in an exclusively disabled cohort of patients. It may provide additional information to assist clinicians and patients in ACP.

The average age of $62.5 \mathrm{yr}$ at time of sudden death was comparable with the literature for individuals who sustain an in-hospital sudden death [8]. The higher prevalence of cervical SCIs in this study is consistent with the overall higher prevalence of tetraplegic injuries versus paraplegic injuries [11]. While a correlation between BMI and poor survivability has been demonstrated in a large, nondisabled population sample [12], there was no association in this SCI cohort. However, the changes in body composition, particularly muscle mass, that occur after SCI can lead to an underestimation of obesity by BMI methods [12]. Additionally, height records in the study population institution are not measured but rather taken from the patient's history, because accurate height recordings are difficult to obtain as a result of joint contractures and/or spasticity. Thus, these BMI measurements are inherently prone to error.

Certain comorbidities in the SCI population may decrease the prognosis of survival after cardiopulmonary arrest. Chronic kidney disease is an established risk factor for sudden cardiac arrest [13]. Patients with SCI are at increased risk for chronic kidney disease and equations used in the nondisabled population to determine estimated glomerular filtration rate may overestimate kidney function in the SCI population [14]. This is attributed to 
poor accounting for muscle mass, impacted by SCIrelated muscle atrophy, especially in patients with motor complete injuries (present in $94.4 \%$ of this cohort). Silent cardiac ischemia is another concern in the SCI population. Individuals with SCI rostral to thoracic 4 may not recognize cardiac angina pain due to disruption of sympathetic afferent fibers traveling with cardiac pain fibers. In this study, nearly 80 percent of the patients had motor and sensory complete injuries. Interestingly, one of the two survivors was classified as having cervical 5 motor and sensory complete tetraplegia. Finally, although not unique to the SCI population, grade III and grade IV pressure ulcers are associated with increased mortality and pressure ulcer management was the most common reason for admission in this study [15].

End-of-life discussions are crucial in any patient population. Although we found no specific contributing factors to patients' survivability after cardiopulmonary resuscitation, this study suggests that the SCI patient population has a poorer survival rate after CPR than general hospitalized patient populations. Having this knowledge may encourage SCI physicians to be more aggressive about discussing advanced directives with their patients, especially patients at risk of significantly decreased functional status after a CPR event. In addition, patients may have a strong desire to not die in the ICU on a ventilator. Armed with this information, patients may be more inclined to decline CPR.

This study has several limitations that affect generalizability. The data were from Veterans, who may present a unique cohort. Additionally, all subjects were male and the relevance to females with SCI is unknown. This investigation was based at a tertiary SCI center that offers aggressive treatments for acute and chronic medical illness, thereby attracting patients who are potentially more medically complex than the typical community-dwelling individual with SCI. Importantly, the small sample size did not afford the ability to validly subanalyze the cohort to assess factors that influenced outcome from CPR and limited statistical analysis. Despite these limitations, this pilot study provides clinicians with survival rates after CPR in the SCI population that can help guide ACP with patients and families.

\section{CONCLUSIONS}

The purpose of this pilot study was to better characterize the prevalence of sudden in-hospital death and the survivability after CPR in the SCI population. Our findings, although limited by sample size, single location, and specialty population, suggest that survivability after CPR is likely equally as poor as for patients with other neurological conditions. We hope that these findings will help guide further inquiry into defining the appropriate circumstances for resuscitative efforts in the SCI population.

\section{ACKNOWLEDGMENTS}

\section{Author Contributions:}

Study concept and design: D. Caruso, D. X. Cifu. Acquisition of data: D. Caruso, W. E. Carter. Drafting of manuscript: D. Caruso, W. E. Carter. Statistical analysis: W. Carne.

Financial Disclosures: The authors have declared that no competing interests exist.

Funding/Support: This material was unfunded at the time of manuscript preparation.

Institutional Review: The study protocol was approved by the local institutional Internal Review Board.

\section{REFERENCES}

1. Standards and guidelines for cardiopulmonary resuscitation (CPR) and emergency cardiac care (ECC). JAMA. 1980;244(5):453-509. [PMID:6104736] http://dx.doi.org/10.1001/jama.1980.03310050017015

2. Sandroni C, Nolan J, Cavallaro F, Antonelli M. In-hospital cardiac arrest: Incidence, prognosis and possible measures to improve survival. Intensive Care Med. 2007; 33(2): 237-45. [PMID:17019558] http://dx.doi.org/10.1007/s00134-006-0326-z

3. Adams DH, Snedden DP. How misconceptions among elderly patients regarding survival outcomes of inpatient cardiopulmonary resuscitation affect do-not-resuscitate orders. J Am Osteopath Assoc. 2006;106(7):402-4. [PMID:16912338]

4. Wenger NS, Phillips RS, Teno JM, Oye RK, Dawson NV, Liu H, Califf R, Layde P, Hakim R, Lynn J. Physician understanding of patient resuscitation preferences: Insights and clinical implications. J Am Geriatr Soc. 2000;48(5, Suppl):S44-51. [PMID:10809456]

5. Moss AH. Informing the patient about cardiopulmonary resuscitation: When the risks outweigh the benefits. J Gen 
Intern Med. 1989;4(4):349-55. [PMID:2668460]

http://dx.doi.org/10.1007/BF02597409

6. Fugate JE, Brinjikji W, Mandrekar JN, Cloft HJ, White RD, Wijdicks EF, Rabinstein AA. Post-cardiac arrest mortality is declining: A study of the US National Inpatient Sample 2001 to 2009. Circulation. 2012;126(5):546-50. [PMID:22740113] http://dx.doi.org/10.1161/CIRCULATIONAHA.111.088807

7. Ebell MH, Smith M, Kruse JA, Drader-Wilcox J, Novak J. Effect of race on survival following in-hospital cardiopulmonary resuscitation. J Fam Pract. 1995;40(6):571-77. [PMID:7775911]

8. Jain R, Nallamothu BK, Chan PS; American Heart Association National Registry of Cardiopulmonary Resuscitation (NRCPR) investigators. Body mass index and survival after in-hospital cardiac arrest. Circ Cardiovasc Qual Outcomes. 2010;3(5):490-97. [PMID:20699443] http://dx.doi.org/10.1161/CIRCOUTCOMES.109.912501

9. Gershengorn HB, Li G, Kramer A, Wunsch H. Survival and functional outcomes after cardiopulmonary resuscitation in the intensive care unit. J Crit Care. 2012;27(4): 421.e9-17. [PMID:22227081] http://dx.doi.org/10.1016/j.jcrc.2011.11.001

10. Dolinak D, Balraj E. Autonomic dysreflexia and sudden death in people with traumatic spinal cord injury. Am J Forensic Med Pathol. 2007;28(2):95-98.

[PMID:17525555] http://dx.doi.org/10.1097/PAF.0b013e3180600f99

11. National Spinal Cord Injury Statistical Center. Facts and figures at a glance [Internet]. Birmingham (AL): University of Alabama at Birmingham; 2013 Mar. Available from: https://www.nscisc.uab.edu/PublicDocuments/ fact_figures_docs/Facts\%202013.pdf

12. Eriks-Hoogland I, Hilfiker R, Baumberger M, Balk S, Stucki G, Perret C. Clinical assessment of obesity in persons with spinal cord injury: Validity of waist circumfer- ence, body mass index, and anthropometric index. J Spinal Cord Med. 2011;34(4):416-22. [PMID:21903016] http://dx.doi.org/10.1179/2045772311Y.0000000014

13. Whitman IR, Feldman HI, Deo R. CKD and sudden cardiac death: Epidemiology, mechanisms, and therapeutic approaches. J Am Soc Nephrol. 2012;23(12):1929-39. [PMID:23100219] http://dx.doi.org/10.1681/ASN.2012010037

14. Fischer MJ, Krishnamoorthi VR, Smith BM, Evans CT, St Andre JR, Ganesh S, Huo Z, Stroupe KT. Prevalence of chronic kidney disease in patients with spinal cord injuries/disorders. Am J Nephrol. 2012;36(6):542-48.

[PMID:23221005] http://dx.doi.org/10.1159/000345460

15. Cao Y, Krause JS, DiPiro N. Risk factors for mortality after spinal cord injury in the USA. Spinal Cord. 2013; 51(5):413-18. [PMID:23380680] http://dx.doi.org/10.1038/sc.2013.2

Submitted for publication May 24, 2013. Accepted in revised form March 25, 2014.

This article and any supplementary material should be cited as follows:

Caruso D, Carter WE, Cifu DX, Carne W. Survival of male patients with spinal cord injury after cardiac arrest in Department of Veterans Affairs hospital: Pilot study. J Rehabil Res Dev. 2014;51(7):1103-8.

http://dx.doi.org/10.1682/JRRD.2013.05.0119

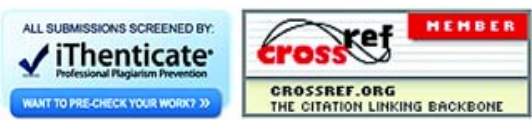

